\title{
Littérature, anthropologie et critique thématique
}

\section{Romano Luperini}

Traducteur : Silvia Disegni

\section{OpenEdition \\ Journals}

Édition électronique

URL : http://journals.openedition.org/recherchestravaux/577

DOI : 10.4000/recherchestravaux.577

ISSN : 1969-6434

\section{Éditeur}

UGA Éditions/Université Grenoble Alpes

\section{Édition imprimée}

Date de publication : 15 mai 2013

Pagination : 29-35

ISBN : 978-2-84310-248-6

ISSN : 0151-1874

\section{Référence électronique}

Romano Luperini, «Littérature, anthropologie et critique thématique », Recherches \& Travaux [En ligne], 82 | 2013, mis en ligne le 15 novembre 2014, consulté le 08 septembre 2020. URL : http:// journals.openedition.org/recherchestravaux/577 ; DOI : https://doi.org/10.4000/recherchestravaux. 577

(C) Recherches \& Travaux 
Romano LUPERINI

Université de Sienne, Italie

\title{
Littérature, anthropologie et critique thématique
}

\author{
(Traduit de l'italien par Silvia Disegni)
}

Depuis un quart de siècle, du moins à partir d'un colloque parisien et d'un numéro spécial de Poétique qui en publiait les actes, Du thème en littérature puis d'un livre édité par Werner Sollors au titre significatif, The Return of Thematic Criticism ${ }^{2}$, la forme la plus commune et la plus fréquente adoptée par l'anthropologie culturelle pour faire son entrée dans le domaine de la critique littéraire est celle de la recherche thématique, qui s'est affirmée sur une grande échelle, surtout dans les études de littérature comparée, en particulier en Amérique du Nord et en Europe. La critique thématique avait fait sa première apparition à la fin du $\mathrm{XIX}^{\mathrm{e}}$ siècle et au début $\mathrm{du} \mathrm{Xx}^{\mathrm{e}}$ siècle au sein de l'école positiviste, qui l'avait prise dans les mailles de ses fiches de lecture érudites. Elle subit ensuite l'opposition théorique de la critique idéaliste, puis de la critique marxiste et structuraliste. Elle réapparaît au cours des deux dernières décennies du $\mathrm{xx}^{\mathrm{e}}$ siècle, forte de la fortune en France des études sur les mentalités et sur la "culture matérielle» inaugurée par l'école des Annales, par la mythographie et la psychanalyse (de Bachelard à Mauron) et, en Amérique du Nord, par les études littéraires des archétypes promues par Frye, mais aussi par l'approche historico-anthropologique présente dans les cultural studies, par ce qu'on appelle le "nouvel historicisme», par les gender studies et, enfin, par les études postcoloniales inaugurées par les enquêtes géniales d'Edward Saïd'. Il ne faut pas oublier non plus l'apport original de l'école russe

I. Poétique, $\mathrm{n}^{\circ} 64, \mathrm{19} 85$, XVI.

2. W. Sollors (dir.), The Return of Thematic Criticism, Cambridge-Londres, Harvard University Press, 1993.

3. E. Saïd, Culture and Imperialism, New York, Knopf, I993; du même, The World, the Text, and the Critic, Cambridge, Harvard University Press, 1983. 
de Bakhtine et de Lotman, dont Ziolkowski et Shcheglov sont les continuateurs actuels.

La critique thématique met fin au refoulement des contenus et de la vie réelle que le formalisme russe avait provoqué. Ce n'est pas un hasard si l'affirmation de la première coïncide avec la crise du second et avec l'avènement de la saison poststructuraliste. Toutefois, la ferveur des études n'a pas été suivie par une mise au point théorique adéquate de la notion de thème. Cesare Segre a signalé à ce propos une certaine "bivalence». Selon cet auteur, la notion de thème oscille en effet entre deux pôles : l'«argument", d'un côté, et l'«idée inspiratrice", de l'autre, autrement dit entre une approche objective et une autre approche fondée sur la subjectivité de l'auteur. En effet, le thème est situé à la fois à l'intérieur de l'œuvre et au cœur de l'expérience et de la culture du lecteur : il fait partie d'un imaginaire largement répandu, aussi bien horizontalement, d'un pays à l'autre, que verticalement, d'un siècle à l'autre. En somme, l'imaginaire constitue un terrain commun qui finit par devenir un véritable pont permettant la communication entre l'œuvre et le lecteur. Si l'œuvre est un grand réservoir de mythes et de symboles, et donc de thèmes, ceux-ci peuvent trouver une correspondance dans la réalité anthropologique et dans l'inconscient collectif des lecteurs. Dans le rapport entre le texte et l'interprète, le médium qui permet à la familiarité de l'emporter sur le sentiment d'étrangeté et de comprendre le message dans toute sa richesse intellectuelle et émotive, ce n'est pas seulement le langage, comme l'affirmait Gadamer : c'est aussi l'imaginaire. C'est de ce point de vue qu'il faut développer cette observation de Segre : «motifs et thèmes sont en somme le langage (quasiment des mots, phrases, schémas syntaxiques) de notre contact cognitif avec le monde de l'homme ${ }^{4}$.

Ceci explique pourquoi la "thématologie» connaît actuellement une telle diffusion en Italie, aussi bien dans le domaine des études comparées que dans celui des études classiques (c'est là que la rencontre entre littératures classiques, mythologie et anthropologie a été particulièrement féconde), et cela dans les différentes branches des études littéraires (du domaine italien en particulier), mais aussi en didactique de la littérature, en particulier dans le secondaire, sous la forme des "parcours thématiques». Une telle diffusion a permis des acquis importants : l'anthropologie, l'ethnologie et la mythologie ont fait irruption au sein d'une discipline qui les avait presque ignorées jusque-là, du moins dans notre pays; les contenus matériels, trop longtemps ignorés ou sous-estimés à cause de l'hégémonie de l'idéalisme et du formalisme, sont

4. C. Segre, "Temi/motivo», dans Avviamento all'analisi del testo letterario, Turin, Einaudi, I985, p. 356 (nous traduisons). 
de nouveau à l'honneur; enfin, un démenti décisif a été infligé à la théorie du caractère autoréférentiel de la littérature, à la primauté du signifiant et au caractère inessentiel de l'élément cognitif. Des notions telles que la mimesis, la connaissance ou la représentation de la réalité ont vu à nouveau le jour en occupant une place fondamentale, en particulier grâce à l'effet parallèle, absolument autonome, de la redécouverte d'un chercheur pourtant étranger à la problématique du contenu thématique, Ludwig Auerbach, et de son chef-d'œuvre, Mimesis, dont on a célébré le cinquantenaire il y a quelques années. Pour ce qui est des études d'italien et de didactique de la littérature italienne, l'importance accordée au thème a permis un classement typologique des œuvres différent de celui qui était établi de manière plus traditionnelle à partir des mouvements littéraires. Il a favorisé l'adoption d'un point de vue comparatiste, mais aussi interdisciplinaire et transdisciplinaire (ouvert à la peinture, à la sculpture, à la musique et au cinéma). En outre, l'étude de la solidarité entre le thème et la forme du contenu s'est révélée précieuse pour l'étude des genres littéraires, compte tenu du lien organique entre une certaine structure formelle et un certain contenu thématique. Dans ce domaine, les études menées par Sergio Zatti sur la poésie du Xvi ${ }^{\mathrm{e}}$ siècle ou sur l'autobiographie sont restées célèbres. Mais je pourrais ajouter, par exemple, le cas du roman d'adultère, dont le succès coïncide avec un type de structure narrative omnisciente ou objective et, malgré tout, avec la forte présence d'un narrateur dont le déclin va cependant de pair avec l'affirmation d'un roman ouvert où domine un "poly-perspectivisme" relativiste et où la femme adultère peut finalement prendre la parole, en racontant sa liaison amoureuse de son propre point de vue. En effet, si l'on assiste, au cœur de la voix narrative du roman d'adultère le plus célèbre du XIX ${ }^{\mathrm{e}}$ siècle, Madame Bovary, à une oscillation, à un double mouvement de participation et de détachement critique qu'Auerbach avait déjà relevé, ce qui domine ensuite, c'est malgré tout une condamnation qui est d'autant plus péremptoire qu'elle n'est jamais explicite, mais perçue comme implicite dans le jugement qui en est donné de manière impersonnelle, comme si elle était l'expression d'un inconscient politique qui n'a même pas besoin d'être porté à la lumière - mais on pourrait dire des choses analogues à propos du Mari d'Hélène de Verga, de La Vertu de Cecchina de Matilde Serao, d'Anna Karénine de Tolstoï ou d'Effi Briest de Fontane. Tout change au début du $\mathrm{xx}^{\mathrm{e}}$ siècle, aussi bien dans la longue nouvelle de Musil intitulée L'Accomplissement de l'amour, que dans la conclusion d'Ulysse de Joyce, confiée, ce qui est significatif, à Molly Bloom : la différence structurelle du récit - désormais ouverte, expérimentale et variable, pour les différentes perspectives choisies - va de pair avec l'avènement du point de vue féminin. En un siècle, l'adultère passe du statut de tragédie sociale au rang 
d'un simple problème psychologique, du statut d'événement public lourd de conséquences éthiques, mais aussi pénales, à celui d'une histoire entièrement personnelle. La conséquence en a été l'effondrement et la dispersion d'un sous-genre narratif, le roman d'adultère, qui avait été prospère pendant de nombreuses années. En somme, le thème et la forme sont liés et l'éclipse du thème entraîne celle de la structure narrative auquel il était associé.

De tels exemples nous permettent également d'aller au plus près des problèmes que la critique anthropologique et thématique pose à la critique. Je les réduirais schématiquement à deux éléments précis : I) le thème est un contenu de réalité extratextuel qui renvoie à une expérience et à un vécu universels; c'est un stéréotype et, pour cette raison, il est universellement humain. Cependant, bien qu'il se répète toujours dans des formes reconnaissables, il s'articule à chaque fois de manière différente au sein de constructions dont chacune est pourvue d'une singularité spécifique; 2) le thème n'exprime pas seulement une concrétion anthropologique de longue durée ou un inconscient collectif à substance intemporelle, il a aussi un contenu gnoséologique : il exprime donc un moment d'autoconscience historique relative à une période donnée. Le fait est que l'imaginaire artistique ne peut être réduit ni à la dialectique nietzschéenne entre apollinien (ou rêve) et dionysiaque (ou ivresse), ni à celle que propose Jung entre «introversion» et «extraversion». On pourrait plutôt dire, avec Eugenio Montale, que l'imaginaire artistique est un rêve qui se produit en présence de la raison : autrement dit, il exprime une forme de médiation entre l'inconscient (privé et collectif) et la culture, entre les archétypes et l'idéologie, ou encore, dirait le psychanalyste Matte Blanco, entre la logique asymétrique et la logique symétrique.

Une telle prise de conscience peut aider le critique littéraire qui utilise des instruments empruntés à une autre discipline (en l'occurrence, l'anthropologie) à éviter les risques qu'implique une telle opération. Par exemple, une certaine approche thématico-anthropologique tend souvent à miser non pas sur un seul texte, mais sur une série d'œuvres caractérisées par la présence d'un même thème, et à se soustraire, de ce fait, à l'analyse du moment génétique de l'œuvre, de sa reconstruction en termes historiques, sociologiques, psychologiques et spécifiquement littéraires; elle tend aussi à ignorer ou à sous-estimer la question du jugement esthétique et de la valeur historicolittéraire, en finissant par considérer les textes comme les documents d'un thème plutôt que comme des monuments ou des exemples pourvus de valeur artistique; en outre, ou par conséquent, elle tend à négliger les aspects formels et littéraires des ouvres considérées; elle finit fatalement par accorder peu d'importance au moment historico-idéologique pour donner la primauté au plan anhistorique de l'inconscient collectif, des archétypes, des symboles, des 
mythes et des rites atemporels. Puis, sur le plan de la didactique de la littérature (en quoi se traduit souvent la critique thématique), elle risque de focaliser l'attention sur des détails secondaires ou sur des motifs à faible teneur historico-anthropologique, au lieu de se concentrer sur les grands thèmes de la condition humaine (Spitzer s'était déjà moqué du choix du cheval comme objet d'étude privilégié).

J'essaierai de traiter ces deux problèmes de manière unitaire : d'ailleurs, l'identité esthétique qui fait d'une œuvre un unicum non réitérable est toujours accompagnée d'une identité historico-littéraire précise. Prenons le thème, cher à l'approche anthropologique, du "différent», de l'"autre», qu'il faut associer pour plusieurs raisons à celui de l'«exclu» ou de l'«exilé». Une telle forme d'asociabilité est également documentée dans le récent Dizionario dei temi letterari ${ }^{5}$, dont toutes les entrées sont liées par un système de renvois favorisant leur constitution en constellation ou en réseau complexe. Chez Verga, le thème de la différence et de l'exclusion revient de manière obsessionnelle du début à la fin de sa production majeure, et cela depuis son premier texte narratif vériste, la nouvelle intitulée "Rosso Malpelo», jusqu'au dernier roman porté à terme du cycle des Vaincus, Mastro-don Gesualdo. Dans le premier, le thème archétypal de l'altérité renvoie au "roux», qui existe en anthropologie et en ethnologie (et qui est également un signal de danger pour l'inconscient collectif); dans le second, à l'idée d'intrusion au sein d'une classe différente (Gesualdo est un manœuvre pour les nobles et un noble pour les manœuvres : il est donc condamné, à une forme d'isolement). Mais la première autant que la deuxième image de la diversité est inséparable de la notion positiviste et darwinienne de lutte pour la vie, de sélection naturelle : elles appartiennent donc toutes les deux à un domaine culturel précis qui exclut l'"autre» également pour des raisons économiques et sociales déterminées par l'histoire. Il est encore plus intéressant de situer dans cette perspective le protagoniste du roman Les Malavoglia. Au cours du XIX ${ }^{\mathrm{e}}$ siècle marqué par le romantisme et le Risorgimento, si le thème de l'exil avait pris, avec le jeune 'Ntoni, une tournure éminemment politique et économique, à partir de Iacopo Ortis de Foscolo et jusqu'à Foi et Beauté de Tommaseo et Confessions d'un Italien de Nievo, il commence à prendre une valeur symbolique et ontologique. Dans le final des Malavoglia, il semble même anticiper un thème typique de la production d'Ungaretti, celui du port enseveli, de l'exclusion du personnage, coupé de ses origines et de ses racines originelles. Une telle articulation est le résultat d'une resémantisation radicale du mot «exil» et du thème implicite.

5. R. Ceserani, M. Domenichelli, P. Fasano (dir.), Dizionario dei temi letterari, Turin, UTET, 3 vol., 2007. 
Elle ne peut être comprise qu'en termes d'histoire. Si Giacomo Debenedetti avait déjà remarqué que l'on assistait avec le personnage de 'Ntoni au retour de l'archétype du «sans terre» et du «sans patrie», de l'exilé condamné à errer pour l'éternité ${ }^{6}$, il faudra bien se demander pourquoi, pour des raisons historiques et dans tout le bassin de la culture moderne, depuis Baudelaire jusqu'à Verga, Ungaretti et au premier Montale (celui de La Farandole des enfants sur la grève), l'artiste ne s'identifie plus à une figure de citoyen patriote luttant pour le bien de la patrie, mais se représente plutôt comme un "exclu», un outcast (dira Montale), une créature depuis toujours dépourvue de racines. De ce point de vue, le "différent» de Verga, l'enfant terrible aux cheveux roux et à la logique fortement démystificatrice qui est pourtant très lié à un moment politique et culturel précis, peut sans doute anticiper symboliquement de nombreux intellectuels modernes, y compris Pasolini.

Si l'on s'arrête sur cet exemple et que l'on analyse le texte, on peut relever la manière spécifiquement littéraire de traiter le thème de l'exil et de l'exclu dans la clôture des Malavoglia. D'ailleurs, la littérature connaît la réalité et la représente figurativement à travers des processus de formalisation précis étroitement liés, de manière intrinsèque, aux contenus thématiques. L’opposition patron 'Ntoni/'Ntoni, vieux/jeune, se développe dans le roman à travers deux idées différentes de la temporalité. 'Ntoni oppose à une conception cyclique, fondée sur l'éternel retour des constellations et des récoltes, et donc typique de l'univers rural archaïque, une autre conception, linéaire et progressive, caractéristique de la modernité qu'il a connue dans les grandes villes. Entre ces deux approches, il y a un saut anthropologique : le grand-père trouve son modèle dans les moineaux qui, chaque année, reviennent faire leur nid sous le même toit, alors que 'Ntoni lui oppose un temps fondé sur le progrès, le développement et la révolution (il arrive même à un certain moment à prêcher une sorte de socialisme). C'est une erreur qu'il paie par une sorte de loi du talion, puisqu'il finit par être condamné à l'exil par ce monde rural archaïque qu'il avait voulu fuir, mais qui lui apparaît désormais comme une sorte de nid ou d'oasis protectrice. En effet, avec son adieu final, il se sépare aussi du retour périodique des constellations, de l'éternel murmure de la mer et du rythme du temps circulaire et ethnologique qui scande les journées des paysans et des pêcheurs. Une telle situation de déracinement et de deuil est indiquée par une série d'éléments formels : la réitération de certains mots et expressions (le verbe «commencer» revient sept fois, dont trois dans la même locution : «commencer la propre journée»; certains syntagmes, comme «les

6. G. Debenedetti, Il romanzo del Novecento : quaderni inediti [1971], Milan, Garzanti, 1996. 
portes fermées» ou "au cœur du village», ou «le village tout noir » réapparaissent constamment, etc.). Signalons aussi le retour des rimes, des assonances et des allitérations; pensons simplement à ce passage : "Tornò a guardare il mare, che sera fatto amaranto, tutto seminato di barche che avevano cominciato la loro giornata anche loro ${ }^{7}$ " ( il revint regarder la mer, qui tournait à l'amarante, entièrement parsemée de barques qui, elles aussi, avaient commencé leur journée»). C'est d'autant plus déchirant que tout cela se réfère à un monde qui est en train de disparaître pour sombrer dans la mémoire. L'usage des temps verbaux est d'ailleurs cohérent. L'imparfait duratif est constamment employé pour décrire la mer, les constellations et le village, alors que l'aoriste, le temps historique de l'action ponctuelle et momentanée, marque tous les mouvements ou toutes les pensées de 'Ntoni. L'opposition passé simple/imparfait marque une autre opposition : celle qui existe entre le moderne et le prémoderne, entre le temps linéaire ou historique et le temps cyclique et ethnographique. L'exclusion du sujet, chassé du monde rassurant de l'éternel retour, sa condamnation à la modernité, la découverte même que le sens de la vie est devant soi et non derrière, dans un monde des origines perdu à jamais, la tragédie de l'exil qui en découle, celle des racines coupées et perdues, sont donc rendues par une série d'artifices formels. Il est évident que si l'on omet de mener une analyse de ce type, on risque de perdre la richesse thématique des références ethnologiques et anthropologiques que le passage entend communiquer. Sans elle, il serait également impossible de comprendre les raisons de sa grandeur esthétique.

La critique thématique et anthropologique est désormais un état de fait. Elle nous enseigne que c'est une erreur de négliger l'ordre matériel des contenus et elle nous induit à tenir compte de l'expérience du vécu. Une telle irruption de concrétude ne peut que produire des effets salutaires, tant sur la critique trop souvent dévoyée par une idée autoréférentielle de la littérature, que sur la didactique de celle-ci. Elle permet d'intéresser les jeunes lecteurs à la richesse de l'expérience accumulée dans les textes (songeons, par exemple, à la manière dont on peut lire la grande saison du roman $\mathrm{du} \mathrm{XVIII}^{\mathrm{e}}$ siècle à nos jours comme une véritable anthropologie de la modernité). Mais, naturellement, tout cela ne peut justifier que l'on réduise la critique littéraire et l'enseignement de la littérature à l'illustration documentaire et subsidiaire d'un thème.

7. G. Verga, I Malavoglia [I88I], Milan, Einaudi, 1985, p. 277. 